EESTI NSV TEȦDUSTE AKÁDEEMIA TOIMETISED. 31. KOIDE FOOSIKA * MATEMAATIKA. 1982, NR. 4

ИЗВЕСТИЯ АКАДЕМИИ НАУК ЭСТОНСКОИ ССР. ТОМ 31 ФНЗНКА * МАТЕМАТИКА, 1982, № 4

О. ВААРМАНН, Марика ЛОМП

удК $517.948: 513.88$

\title{
О РАСШИРЕНИИ ОБЛАСТИ СХОДИМОСТИ МЕТОДОВ С АППРОКСИМАЦИЕЙ ПСЕВДООБРАТНОГО ОПЕРАТОРА
}

\author{
(Представил Н. Алумяэ)
}

Рассмотрим нелинейное уравнение

$$
\left[F^{\prime}(x)\right]^{*} F(x)=0,
$$

где $F(x)$ - непрерывно дифференцируемый оператор, действующий из одного гильбертова пространства $H_{1}$ в другое $H_{2}$, причем обратимость оператора $F^{\prime}(x)$ не обязательна. Для решения уравнения (1) можно привлекать итерационные методы, основанные на псевдообращении, или методы типа Левенберга-Марквардта, описанные, в частности, в $\left[{ }^{1,2}\right]$. Однако эти методы весьма требовательны к качеству начальных приближений. Расширить область сходимости, а иногда и ускорить сходимость методов удается введением т. н. релаксационных параметров, определяющих длину шага итерации.

В настоящей работе рассматриваются итерационные методы вида

$$
x_{k+1}=x_{k}-\varepsilon_{k} A_{k} F\left(x_{k}\right), \quad k=0,1,2, \ldots,
$$

где $A_{k}$ аппроксимирует оператор $\left[F^{\prime}\left(x_{k}\right)\right]^{+}, \varepsilon_{k}-$ релаксационный параметр $\left(0<\varepsilon_{k} \leqslant 1\right)$, подчиняющийся требованиям, сформулированным в приводимых ниже теоремах.

1. Обозначим оператор ортогонального проектирования гильбертова пространства $H$ на его подпространство $L$ через $P_{L}$, область значений оператора $F^{\prime}(x)$ - через $R\left(F^{\prime}(x)\right)$ и его ядро - через $N\left(F^{\prime}(x)\right)$. Для простоты положим $\quad R(x)=R\left(F^{\prime}(x)\right), P_{R(x)}=P_{R\left(F^{\prime}(x)\right)}$ и $P_{k}=P_{R\left(F^{\prime}\left(x_{k}\right)\right) \text {. }}$ Здесь и далее $C, L, L_{0}, L_{1}, N^{\prime}, N_{1}, N, G, \lambda$ и $\Omega$ означают некоторые положительные константы.

Для доказательства теорем нам понадобятся две вспомогательные леммы.

Л ем м а 1. Пусть $S$ - некоторый шар в $H_{1}$ и на $S$ выполнены условия

$$
\begin{array}{ll}
\left\|\left[F^{\prime}(x)\right]^{+}\right\| \leqslant C & \text { для всех } x \in S, \\
\left\|F^{\prime}(x)-F^{\prime}(y)\right\| \leqslant L_{1}\|x-y\| & (x, y \in S),
\end{array}
$$

тогда

$$
\left\|P_{R(x)}-P_{R(y)}\right\| \leqslant L_{0}\|x-y\| .
$$

Доказ а тельст в о. Пусть $H$ - гильбертово пространство, $U$ и $V-$ его замкнутые подпространства, $P_{1}$ и $P_{2}$ - операторы ортогонального проектирования $H$ на $U$ и $V$ соответственно, $P^{(1)}=I-P_{1}$ и $P^{(2)}=I-P_{2}$, где под $I$ понимается единичный оператор в $H$. Тогда $\left(\left[{ }^{3}\right]\right.$, c. $195-$ 197)

$$
\left\|P_{1}-P_{2}\right\|=\max \left\{\sup _{x \in U,\|x\|=1}\left\|P^{(2)}(x)\right\|, \quad \sup _{x \in V,\|x\|=1}\left\|P^{(1)}(x)\right\|\right\} .
$$

Из свойств ортопроектора следует 


$$
\left\|\dot{P}_{R(x)}-\dot{P}_{R(y)}\right\|=\left\|\left(I-\dot{P}_{R(x)}\right)-\left(I-P_{R(y)}\right)\right\|=\left\|\tilde{P}_{N\left(\left[F^{\prime}(x)\right]^{*}\right)}-\dot{P}_{N\left(\left[F^{\prime}(y)\right]^{*}\right)}\right\| .
$$

Полагая $H_{2}=H, N\left(\left[F^{\prime}(x)\right]^{*}\right)=U$ и $N\left(\left[F^{\prime}(y)\right]^{*}\right)=V$, можем записать вектор $u \in U \subset H \quad$ в виде $u=u_{1}+u_{2}$, где $u_{1} \in V=N\left(\left[F^{\prime}(y)\right]^{*}\right)$, $u_{2}=N\left(\left[F^{\prime}(y)\right]^{*}\right)^{\perp}=R\left(I-P_{V}\right)$, и

$$
\begin{aligned}
\sup _{u \in U,\|u\|=1}\left\|\left(I-P_{V}\right) u\right\| & =\sup \left\|u_{2}\right\|==\sup _{u \in U,\|u\|=1}\left\|\left[F^{\prime}(y)\right]^{*+}\left[F^{\prime}(y)\right]^{*} u\right\| \leqslant \\
& \leqslant C \sup _{u \in U,\|u\|=1}\left\|\left[F^{\prime}(y)\right]^{*} u\right\| .
\end{aligned}
$$

Так как $\left[F^{\prime}(x)\right]^{*} u=0$, то

$\sup _{u \in U,\|u\|=1}\left\|\left(I-P_{V}\right) u\right\| \leqslant C \sup _{u \in U,\|u\|=1}\left\|\left(\left[F^{\prime}(y)\right]^{*}-\left[F^{\prime}(x)\right]^{*}\right) u\right\| \leqslant C L_{1}\|x-y\|$.

Аналогично имеем

$$
\sup _{v \in V,\|v\|=1}\left\|\left(I-P_{U}\right) v\right\| \leqslant C L_{1}\|x-y\|
$$

и в качестве $L_{0}$ можем брать $L_{0}=C L_{1}$.

3 а мечани е 1 . Если выполнено условие (4) и

$$
\left\|A_{k}\right\| \leqslant \lambda, \quad k=0,1, \ldots,
$$

то, пользуясь леммой 1 , нетрудно показать, что существуют постоянные $N^{\prime}$ и $N$ такие, что

$$
\left\|\left(P_{R(y)}-P_{R(y)} P_{R(x)}\right) F(x)\right\| \leqslant N^{\prime}\|x-y\| \quad(x, y \in S)
$$

или

$$
\left\|\left(P_{k+1}-P_{k+1} P_{k}\right) F\left(x_{k}\right)\right\| \leqslant N_{k}\left\|P_{k} F\left(x_{k}\right)\right\| \leqslant N\left\|P_{k} F\left(x_{k}\right)\right\|,
$$

где можно положить $N_{k}=\varepsilon_{k} N^{\prime} \lambda$ и $N:=N^{\prime} \dot{\lambda}$.

Л емм а 2. Если ортопроектор $P_{R(x)}$ удовлетворяет в $S$ условию (5) и имеет место соотношение

$$
\left\|\left(P_{R(y)} P_{R(x)} P_{R(y)}-P_{R(y)} P_{R(x)}\right) F(x)\right\| \leqslant N_{2}\|x-y\|^{2},
$$

To

$$
\left\|\left(P_{k+1}-P_{k+1} P_{k}\right) F\left(x_{k}\right)\right\| \leqslant G\left(\varepsilon_{k}\right)\left\|P_{h} F\left(x_{k}\right)\right\|^{2} \leqslant G\left\|P_{k} F\left(x_{k}\right)\right\|^{2},
$$

где

$$
G(\varepsilon)=\varepsilon \lambda L_{0}\left(1+\varepsilon \lambda L_{0} F\right)+\varepsilon^{2} \lambda^{2} N_{2} \cdot u \quad G=G(1), \quad F=\sup _{x \in S}\|F(x)\| .
$$

Доказательство. С учетом соотношения (5) и того, что $\left\|x_{k+1}-x_{k}\right\| \leqslant \varepsilon_{h} \lambda\left\|P_{h} F\left(x_{k}\right)\right\|$, имеем

$$
\left\|P_{k+1} F\left(x_{k}\right)\right\| \leqslant\left\|P_{h} F\left(x_{k}\right)\right\|+\left\|\left(P_{k+1}-P_{k}\right) F\left(x_{k}\right)\right\| \leqslant\left(1+\varepsilon_{k} L_{0} \lambda\right)\left\|P_{k} F\left(x_{k}\right)\right\| .
$$

Далее

$$
\begin{aligned}
& \left\|\left(P_{k+1}-P_{k+1} P_{k}\right) F\left(x_{k}\right)\right\| \leqslant\left\|P_{h+1}\left(P_{k+1}-P_{k}\right) P_{k+1} F\left(x_{k}\right)\right\|+ \\
+ & \left\|\left(P_{k+1} P_{k} P_{k+1}-P_{k+1} P_{k}\right) F\left(x_{k}\right)\right\| \leqslant L_{0}\left\|x_{k+1}-x_{k}\right\|\left\|P_{k} F\left(x_{k}\right)\right\|+ \\
+ & N_{2}\left\|x_{k+1}-x_{k}\right\|^{2} \leqslant G\left(\varepsilon_{k}\right)\left\|P_{h} F\left(x_{k}\right)\right\|^{2} \leqslant G\left\|P_{k} F\left(x_{k}\right)\right\|^{2} .
\end{aligned}
$$

Очевидно, что в случае коммутативности $P_{R(x)}$ и $P_{R(y)}$ соотношение (9) выполняется при $N_{2}=0$.

2. Допустим, что $A_{k}=A_{k} P_{k},\left\|A_{k}\right\| \leqslant \lambda<\infty$ и введем числовые последовательности $\left\{\gamma_{k}\left(\varepsilon_{k}\right)\right\}$, удовлетворяющие условию 


$$
\left\|\hat{P}_{k}-\varepsilon_{k} F^{\prime}\left(x_{k}\right) A_{k}\right\| \leqslant \gamma_{k}\left(\varepsilon_{k}\right),
$$

и при $\varepsilon_{k}=1$ положим $\gamma_{k}=\gamma_{k}(1)$.

Теорема 1. Пусть $x_{0} \in H_{1}, S=\left\{x \in H_{1}:\left\|x-x_{0}\right\| \leqslant \varrho\right.$ u на $S$ выполнены условия:

1. Оператор $F(x)$ дифференцируем (по Фреше).

2. Производная $F^{\prime}(x)$ удовлетворяет условию Липшица

$$
\left\|F^{\prime}(x)-F^{\prime}(y)\right\| \leqslant L_{1}\|x-y\| .
$$

3. $\delta<1$.

4. $0<\varepsilon_{0} \leqslant \varepsilon_{k-1} \leqslant \varepsilon_{k} \leqslant \min \left\{1, \min \varepsilon_{h-1} \delta_{k-1}^{-1 / 2}\right\}$.

Тогда: А. Если выполнено условие (3), $\gamma_{k} \leqslant \gamma<1$ и $r=$ $=\lambda\left\|P_{0} F\left(x_{0}\right)\right\| /(1-\delta) \leqslant \varrho, \quad$ где $\delta=\delta_{0}=1-\varepsilon_{0}+\varepsilon_{0}\left(\gamma_{0}+N\right)+\frac{1}{2} \varepsilon_{0}^{2} \lambda^{2} L_{1} X$ $\times\left\|P_{0} F\left(x_{0}\right)\right\|$, то уравнение (1) имеет в $S$ решение $x^{*}, \kappa$ которому сходится последовательность (2), причем $\left\|x^{*}-x_{0}\right\| \leqslant r_{1} u$

$$
\left\|x_{k}-x^{*}\right\| \leqslant r_{1} \delta^{k} \text {. }
$$

Б. Если $F(x)$ удовлетворяет условию $(10), \gamma \geqslant \gamma_{0} \geqslant \ldots \geqslant \gamma_{k} \geqslant \ldots \geqslant 0$, то последовательность (2) сходится $\kappa x^{*}$ со сверхлинейной скоростью, причем

$$
\left\|x_{k}-x^{*}\right\| \leqslant r_{1} \prod_{i=0}^{h-1} \delta_{i} \quad(k \geqslant 1),
$$

где $\delta_{i}=1-\varepsilon_{i}+\varepsilon_{i} \gamma_{i}+\left(G+\frac{1}{2} \varepsilon_{i}{ }^{2} \lambda^{2} L_{1}\right)\left\|P_{i} F\left(x_{i}\right)\right\| u \delta=\delta_{0}$.

Доказательство. Обозначим $\varphi_{k}(\varepsilon)=\varepsilon_{k} \psi_{k}+1-\varepsilon_{k}$, где $\psi_{k}-$ вещественные числа. Если $\gamma_{k} \leqslant \gamma<1$ (для всех $k=0,1, \ldots$ ), то $\varphi_{k}^{\prime}(\varepsilon)=\gamma_{k}-1 \leqslant \gamma-1<0$ и, следовательно, $\varphi_{k}(\varepsilon)$ является убывающей функцией от $\varepsilon$.

С учетом того, что $\left\|P_{k}-\varepsilon_{k} F^{\prime}\left(x_{k}\right) A_{k}\right\| \leqslant \varepsilon_{k}\left\|P_{k}-F^{\prime}\left(x_{k}\right) A_{k}\right\|+1-\varepsilon_{k} \leqslant$ $\leqslant \varepsilon_{k} \gamma_{k}+1-\varepsilon_{k}, \quad$ в качестве $\gamma_{k}(\varepsilon)$ можем брать $\quad \gamma_{k}\left(\varepsilon_{k}\right)=\varepsilon_{k} \gamma_{k}+1-\varepsilon_{k}$.

По формуле Тейлора

$$
\begin{gathered}
P_{k+1} F\left(x_{k+1}\right)=\left(P_{k+1}-P_{k+1} P_{k}\right) F\left(x_{k}\right)+P_{k+1}\left\{\left(P_{k}-\varepsilon_{k} F^{\prime}\left(x_{k}\right) A_{k}\right) F\left(x_{k}\right)+\right. \\
\left.+\varepsilon_{k} \int_{0}^{1}\left[F^{\prime}\left(x_{k}\right)-F^{\prime}\left(x_{k}+t\left(x_{k+1}-x_{k}\right)\right)\right] A_{k} F\left(x_{k}\right) d t\right\} .
\end{gathered}
$$

A. На основе замечания 1 получаем, что $\left\|P_{k+1} F\left(x_{k+1}\right)\right\| \leqslant \delta_{k}\left\|P_{k} F\left(x_{k}\right)\right\|$, где $\delta_{k}=\gamma_{k}\left(\varepsilon_{k}\right)+\varepsilon_{k} N_{1}+\frac{1}{2} \varepsilon_{k}^{2} \lambda L_{1}\left\|P_{k} F\left(x_{k}\right)\right\|$. Допустим, что $\delta_{k} \leqslant \delta_{0}$. Тогда в силу неравенств $\varepsilon_{k+1}^{2} \delta_{k} \leqslant \varepsilon_{k}^{2}$ и $\left\|P_{k+1} F\left(x_{k+1}\right)\right\| \leqslant \delta_{k}\left\|P_{k} F\left(x_{k}\right)\right\|$ имеем $\varepsilon_{k+1}^{2} \lambda^{2} L_{1}\left\|P_{k+1} F\left(x_{k+1}\right)\right\| \leqslant \varepsilon_{k+1}^{2} \delta_{k} \lambda^{2} L_{1}\left\|P_{k} F\left(x_{k}\right)\right\| \leqslant \varepsilon_{k}^{2} \lambda^{2} L_{1}\left\|P_{k} F\left(x_{k}\right)\right\|$.

Полагая $\psi_{k}=\gamma_{k}+N$, из условия $\delta_{0}<1$ получаем, что $\psi_{k+1} \leqslant \psi_{0}<1$. Поэтому $\varphi_{k+1}\left(\varepsilon_{k+1}\right) \leqslant \varphi_{k+1}\left(\varepsilon_{0}\right) \leqslant \varphi_{0}\left(\varepsilon_{0}\right)$ и $\delta_{k+1}=\varphi_{h+1}\left(\varepsilon_{k+1}\right)+\frac{1}{2} \varepsilon_{h+1}^{2} \lambda^{2} L_{1}\left\|P_{k+1} F\left(x_{k+1}\right)\right\| \leqslant \ldots \leqslant \varphi_{0}\left(\varepsilon_{0}\right)+\frac{1}{2} \varepsilon_{0}^{2} \lambda^{2} L_{1} \times$ $X\left\|P_{0} F\left(x_{0}\right)\right\|=\delta_{0}$.

Б. Если выполнено условие (10), то с помощью (12) получим 


$$
\delta_{k}=\gamma_{k}\left(\varepsilon_{k}\right)+\left(G\left(\varepsilon_{k}\right)+\frac{1}{2} \varepsilon_{k}^{2} \lambda^{2} L_{1}\right)\left\|P_{k} F\left(x_{k}\right)\right\| .
$$

Здесь $\varphi_{k}(\varepsilon)=\gamma_{k}(\varepsilon)$ и поэтому

$$
\gamma_{k+1}\left(\varepsilon_{k+1}\right) \leqslant \gamma_{k+1}\left(\varepsilon_{k}\right)=\varepsilon_{k} \gamma_{k+1}+1-\varepsilon_{k} \leqslant \varepsilon_{k} \gamma_{k}+1-\varepsilon_{k}=\gamma_{k}\left(\varepsilon_{k}\right) .
$$

Далее тем же методом индукции убеждаемся, что при $\varepsilon^{2}{ }_{k+1} \delta_{k} \leqslant \varepsilon_{k}{ }^{2}$ или $\varepsilon_{k+1}=\varepsilon_{k}$ справедливы соотношения $\delta_{k+1} \leqslant \delta_{k} \leqslant \ldots \leqslant \delta_{0}$, причем $\gamma_{k} \rightarrow 0$ при $k \rightarrow \infty$ влечет за собой $\delta_{k} \rightarrow 0$.

В остальном доказательство этой теоремы повторяет с необходимыми изменениями доказательство теоремы 1 из $\left[{ }^{2}\right]$.

3 амечание 2. Если выполнены условия теоремы 1, то условие $\delta=\delta_{0}<1$ можно в случаях А и Б переписать соответственно в виде

$$
\begin{gathered}
\varepsilon_{0}\left(\gamma_{0}+N+\frac{1}{2} \varepsilon_{0} \lambda^{2} L_{1}\left\|P_{0} F\left(x_{0}\right)\right\|\right) \leqslant \varepsilon_{0}, \\
\varepsilon_{0}\left[\gamma_{0}+\left(G+\frac{1}{2} \varepsilon_{0} \lambda^{2} L_{1}\right)\left\|P_{0} F\left(x_{0}\right)\right\|\right]<\varepsilon_{0} .
\end{gathered}
$$

Таким образом, при $\gamma_{0}+N<1 \quad\left(\gamma_{0}-G\left\|P_{0} F\left(x_{0}\right)\right\|<1\right.$ (см. замечание 4)) за счет уменьшения параметра $\varepsilon_{0}$ можно добиться выполнимости условия

$\gamma_{0}+N+\frac{1}{2} \varepsilon_{0} \lambda^{2} L_{1}\left\|P_{0} F\left(x_{0}\right)\right\|<1 \quad\left(\gamma_{0}+\left(G+\frac{1}{2} \varepsilon_{0} \lambda^{2} L_{1}\right)\left\|P_{0} F\left(x_{0}\right)\right\|<1\right)$ для любого $x_{0} \in S$ и, тем самым, выполнимости условия $\delta=\delta_{0}<1$.

3 а мечани е 3 . В теореме $1\left[{ }^{4}\right]$ при вписывании формул допущена ошибка: вместо $\left\|P_{k}-F^{\prime}\left(x_{k}\right) A_{k}\right\| \leqslant \gamma_{k}$ должно быть $\left\|P_{k}-\varepsilon_{k} F^{\prime}\left(x_{k}\right) A_{k}\right\| \leqslant$ $\leqslant \gamma_{k}\left(\varepsilon_{k}\right)=\gamma_{k}$, как в теореме $2\left[{ }^{5}\right]$, на которую опирается доказательство теоремы 1 из $\left[{ }^{4}\right]$.

3. Пусть

$$
\begin{aligned}
& {\left[F^{\prime}(x)\right]^{*} F^{\prime}(x)=B(x), \quad B(x)-\alpha I=M(x, \alpha), \quad M\left(x_{k}, \alpha_{k}\right)=M_{k},} \\
& M^{-1}(x, \alpha)\left[F^{\prime}(x)\right]^{*}=L(x, \alpha) .
\end{aligned}
$$

Рассмотрим итерационный метод

$$
x_{k+1}=x_{k}-\varepsilon_{h} A_{h} F\left(x_{k}\right), \quad k=0,1, \ldots,
$$

где $A_{k}=D_{k}\left[F^{\prime}\left(x_{k}\right)\right]^{*}$ и $D_{k}$ - некоторая аппроксимация оператора $M_{k}^{-1}$. Допустим, что $K$ и $\mu_{k}(k=0,1, \ldots)$ - некоторые (ограниченные) постоянные, удовлетворяющие в $S$ условиям

$$
\left\|F^{\prime}(x)\right\| \leqslant K, \quad\left\|I-M_{k} D_{k}\right\| \leqslant \mu_{k},
$$

и положим $\omega_{k}=\mu_{k} / \alpha_{k}$.

Теорем а 2. Пусть, $x_{0} \in H_{1}, S=\left\{x \in H_{1}:\left\|x-x_{0}\right\| \leqslant \mathrm{Q}\right\}$ ина $S$ выполнены условия $1-4$ теоремь 1.

Тогда: А. Если выполнено условие (3), $\quad \alpha_{k} \leqslant \alpha_{0}, \omega_{k} \leqslant \omega_{0}\left(\alpha_{0}, \omega_{0}<\infty\right)$ u $r_{1}=\lambda\left\|P_{0} F\left(x_{0}\right)\right\| /(1-\delta) \leqslant \mathrm{Q}$, где $\delta=\delta_{0}=1-\varepsilon_{0}+\varepsilon_{0}\left(N+\alpha_{0} K C^{3}+\omega_{0} K^{2}\right)+$ $+\frac{1}{2} \varepsilon_{0}^{2} \lambda^{2} L_{1}\left\|P_{0} F\left(x_{0}\right)\right\|$, то уравнение (1) имеет в $S$ решение $x^{*}, \kappa$ которому сходится последовательность (14), причем $\left\|x^{*}-x_{0}\right\| \leqslant r_{1} u$

$$
\left\|x_{k}-x^{*}\right\| \leqslant r_{1} \delta^{k} .
$$

Б. Если $F(x)$ удовлетворяет условию $(10), n \xi^{k} \leqslant \alpha_{k} \leqslant m \xi^{k} \quad\left(m, n^{\prime}<\infty\right)$, 
$\xi<1, \omega_{k} \leqslant s l^{k}, s>0, l<1$, то последовательность (14) сходится $\kappa x^{*}$ со сверхлинейной скоростью, причем

$$
\left\|x_{k}-x^{*}\right\| \leqslant r_{1} \prod_{i=0}^{k-1} \delta_{i} \quad(k \geqslant 1)
$$

где $\delta_{i}=\left(1-\varepsilon_{i}\right)+\varepsilon_{i}\left(m \xi^{i} K C^{3}+s l^{i} K^{2}\right)+\left(G+\frac{1}{2} \varepsilon_{i}^{2} \lambda^{2} L_{1}\right)\left\|P_{i} F\left(x_{i}\right)\right\| \rightarrow 0 \quad$ npu $i \rightarrow \infty u \delta=\delta_{0}$.

Доказ а тельст во. Так как $\left[{ }^{1,6}\right]$

$$
\left\|L\left(x_{k}, \alpha_{k}\right)\right\| \leqslant\left\|\left[F^{\prime}\left(x_{k}\right)\right]^{+}\right\|, \quad\left\|\left(B_{k}+\alpha_{k} I\right)^{-1}\right\| \leqslant \alpha_{k}^{-1},
$$

To

$$
\begin{aligned}
& \left\|\left[F^{\prime}\left(x_{k}\right)\right]^{+}-L\left(x_{k}, \alpha_{k}\right)\right\| \leqslant \alpha_{k} C^{3}, \\
& \left\|L\left(x_{k}, \alpha_{k}\right)-A_{k}\right\| \leqslant\left\|\left(B_{k}+\alpha_{k} I\right)^{-1}\left(I-M_{k} D_{k}\right)\left[F^{\prime}\left(x_{k}\right)\right]^{*}\right\| \leqslant \omega_{k} K, \\
& \left\|A_{k}\right\|=\left\|\left[F^{\prime}\left(x_{k}\right)\right]^{+}-L\left(x_{k}, \alpha_{k}\right)+L\left(x_{k}, \alpha_{k}\right)-A_{k}-\left[F^{\prime}\left(x_{k}\right)\right]^{+}\right\| \leqslant \\
& \quad \leqslant C+\alpha_{k} C^{3}+\omega_{k} K, \\
& \left\|P_{k}-F^{\prime}\left(x_{k}\right) A_{k}\right\|=\left\|F^{\prime}\left(x_{k}\right)\left(\left[F^{\prime}\left(x_{k}\right)\right]^{+}-A_{k}\right)\right\| \leqslant \alpha_{k} K C^{3}+\omega_{k} K^{2} .
\end{aligned}
$$

Учитывая, что $n \xi^{k} \leqslant \alpha_{k} \leqslant m \xi^{k}$, в качестве $\lambda$ и $\gamma_{k}(k:=0,1, \ldots)$ можем брать

$$
\lambda=C+m C^{3}+\left(\mu_{0} / \alpha_{0}\right) K,
$$

а в случаях А и Б $\gamma_{k}=\alpha_{k} K C^{3}+\omega_{k} K^{2}$ и $\gamma_{k}=m \xi^{k} K C^{3}+s l^{k} K$ соответственно. Существование постоянного $K$ такого, что $\left\|F^{\prime}(x)\right\| \leqslant K$, следует из 2-го условия теоремы, а из 3-го условия вытекает, что $\varphi_{k+1} \leqslant \varphi_{0}<1$.

Так как $\left\|I-M_{k} D_{k}\right\| \leqslant \mu_{k}<1$ и $\lim _{\alpha_{k} \rightarrow 0} M_{k}^{-1}\left[F^{\prime}\left(x_{k}\right)\right]^{*}=\left[F^{\prime}\left(x_{k}\right)\right]^{+}$; то $A_{k}=D_{k}\left[F^{\prime}\left(x_{k}\right)\right]^{*}$ аппроксимирует оператор $\left[F^{\prime}\left(x_{k}\right)\right]^{+}$и, следовательно, теорема 1 применима.

При $\varepsilon_{k}=1$ можно указать условия квадратичной скорости сходимости последовательности $\left\{x_{k}\right\}$, порождаемой методом (14) (ср. $\left.\left[{ }^{1,7}\right]\right)$. Имеет место следующая

Теорема 3. Пусть $x_{0} \in H_{1}, \quad S:=\left\{x \in H_{1}:\left\|x-x_{0}\right\| \leqslant \rho\right\}$ и на $S$ выполнены условия $1-3$ теоремы 1 .

Тогда: А. Если выполнено условие (3), $\alpha_{k} \leqslant \alpha_{0}, \omega_{k} \leqslant \omega_{0}\left(\alpha_{0}, \omega_{0}<\infty\right)$ $u \quad r_{1}=\lambda\left\|P_{0} F\left(x_{0}\right)\right\| /(1-\delta) \leqslant \varrho, \quad$ ¿de $\delta=\delta_{0}=N+\alpha_{0} K C^{3}+\omega_{0} K^{2}+$ $+\frac{1}{2} \lambda^{2} L_{1}\left\|P_{0} F\left(x_{0}\right)\right\|$, то уравнение (1) имеет в $S$ решение $x^{*}, \kappa$ которому сходится последовательность (14), причем $\left\|x^{*}-x_{0}\right\| \leqslant r_{1} u$

$$
\left\|x_{k}-x^{*}\right\| \leqslant r_{1} \delta^{k} \text {. }
$$

Б. Если $F(x)$ удовлетворяет условию $(8), \quad \alpha_{0} \geqslant \alpha_{1} \geqslant \ldots \geqslant \alpha_{k} \geqslant \ldots \geqslant 0$, $\omega_{0} \geqslant \ldots \geqslant \omega_{k} \geqslant \ldots \geqslant 0$, то последовательность (14) сходится $\kappa x^{*}$ со сверхлинейной скоростью, причем

$$
\left\|x_{k}-x^{*}\right\| \leqslant r_{1} \prod_{i=0}^{k-1} \delta_{i} \quad(k \geqslant 1),
$$

где $\delta_{i}=\alpha_{i} K C^{3} f \omega_{i} K^{2}+\frac{1}{2} \lambda^{2} L\left\|P_{0} F\left(x_{0}\right)\right\| \rightarrow 0$ npu $i \rightarrow \infty \quad u \quad \delta=\delta_{0}$.

В. Если $F(x)$ удовлетворяет условию (8), $\alpha_{k} \leqslant m\left\|P_{k} F\left(x_{k}\right)\right\|$, $\omega_{k} \leqslant \Omega\left\|P_{k} F\left(x_{k}\right)\right\|, \quad r_{2}=\lambda H_{0}(\delta) / d \leqslant \varrho, \quad$ əде $\quad H_{k}(\delta)=\sum_{i=k}^{\infty} \delta^{2^{t}}, \quad \delta=\delta_{0}=$ 
$=d\left\|P_{0} F\left(x_{0}\right)\right\| u d=m K C^{3}+\Omega_{0} K^{2}+\frac{1}{2} L_{1} \lambda^{2}+G, \quad$ то уравнение (1) имеет в $S$ решение $x^{*}, \kappa$ которому сходится последовательность (14), причем $\left\|x^{*}-x_{0}\right\| \leqslant r_{2} u$

$$
\left\|x_{k}-x^{*}\right\| \leqslant \lambda H_{k}(\delta) / d .
$$

Доказательство этой теоремы с привлечением полученных здесь и в $\left[{ }^{1}\right]$ результатов не представляет труда.

3 а меч а н и е 4 . Условия $\omega_{k} \leqslant \Omega_{0}\left\|P_{k} F\left(x_{k}\right)\right\|$ и $\alpha_{k} \leqslant m\left\|P_{k} F\left(x_{k}\right)\right\|$ влекут за собой условие $\mu_{k}=0\left(\left\|P_{k} F\left(x_{k}\right)\right\|^{2}\right)$. Если $R\left(F^{\prime}\left(x_{k}\right)\right) \supseteq R\left(F^{\prime}\left(x_{k+1}\right)\right)$, то $G=0$, в частности, при $R\left(F^{\prime}(x)\right)=H_{2}$.

4. Выведенные выше оценки скорости сходимости являются в сущности оценками погрешности методов, привлекающих для вычисления обобщенного решения псевдообращение, при условии, что не все необходимые вычисления производятся точно, а при вычислении оператора $F^{\prime}(x)$ или решении соответствующих линейных уравнений может быть обеспечена лишь некоторая степень точности.

Таким образом, теоремы $1-3$ дают условия сходимости и устойчивости методов, использующих псевдообращение, при возмущенных исходных операторах $F^{\prime}(x)$ или $M(x, \alpha)$. Отметим еще, что полученные чисто теоретические условия относительно величины шага указывают на возможность ослабления требований к качеству начальных приближений $x_{0}$ и $A_{0}$.

Приме р 1. Решить систему

$$
F(x)=\left\{\begin{array}{l}
f_{1}\left(x_{1}, x_{2}\right)=x_{1}^{2}+x_{2}{ }^{2}-2=0, \\
f_{2}\left(x_{1}, x_{2}\right)=x_{1}-x_{2}=0, \\
f_{3}\left(x_{1}, x_{2}\right)=x_{1} x_{2}-1=0,
\end{array}\right.
$$

имеющую нулевые точки $(1,1)$ и $(-1,-1)$.

При ме р 2. Решить систему

$$
F(x)=\left\{\begin{array}{l}
f_{1}\left(x_{1}, x_{2}\right)=x_{1}^{2}+x_{2}{ }^{2}-2=0, \\
f_{2}\left(x_{1}, x_{2}\right)=\left(x_{1}-2\right)^{2}+x_{2}^{2}-2=0, \\
f_{3}\left(x_{1}, x_{2}\right)=\left(x_{1}-1\right)^{2}+x_{2}^{2}-9=0,
\end{array}\right.
$$

имеющую $\min _{x} \sum_{i=1}^{3} f_{i}{ }^{2}=42,6666$ в точке $(1 ; \pm 1,914854)$.

Пример 3. Решить систему

$$
f_{i}\left(x_{1}, x_{2}\right)=y_{i}-x_{1}-x_{2} s_{i}, \quad i=1,2, \ldots, 25 .
$$

Приме р 4. Решить систему

$$
f_{i}\left(x_{1}, x_{2}\right)=y_{i}-x_{1} s_{i}^{2}, \quad i=1,2, \ldots, 25 .
$$

Пример 5. Решить систему

$$
f_{i}\left(x_{1}, x_{2}\right)=y_{i}-x_{1} \mathrm{e}^{x_{1} s_{t}}, \quad i=1,2, \ldots, 25 .
$$

Данные для $y_{i}$ и $s_{i}$ в примерах $3-5$ заимствованы из $\left[{ }^{8}\right]$.

Для решения этих систем используем итерационный метод вида

$$
x_{k+1}=x_{k}-\varepsilon_{k} A_{k} F\left(x_{k}\right),
$$

где

$$
\hat{A_{k}}=\left[B\left(x_{k}\right)+\alpha_{k} I\right]^{-1}\left[F^{\prime}\left(x_{k}\right)\right]^{*}, \quad B\left(x_{k}\right)=\left[F^{\prime}\left(x_{k}\right)\right]^{*} F^{\prime}\left(x_{k}\right),
$$

$\alpha_{k}$ - последовательность положительных чисел, причем $\alpha_{k} \rightarrow 0$ при $k \rightarrow \infty$. 
Пронумеруем итерационные процессы в следующем порядке:

1. $\varepsilon_{k}=1, \alpha_{k}=0, \kappa=0,1,2, \ldots$.

2. $\varepsilon_{k}=1, \alpha_{k}=\xi \Sigma_{0}, 0<\xi<1, \kappa=0,1,2, \ldots$, где $\sum_{k}=\max _{i} \sum_{j=1}^{n}\left|b_{i j}^{(k)}\right|$, а $b_{i j}, i, j=1, \ldots, n,-$ элементы матрицы $B\left(x_{k}\right)$. Здесь $\xi=0,001$.

3. $\varepsilon_{k}=1, \alpha_{k}=\xi \cdot \Sigma_{k}, k=0,1,2, \ldots$.

4. $\varepsilon_{k}=1, \alpha_{k}$ выбираем, как в $\left[{ }^{9}\right]$.

5. $\alpha_{k}=0$ и $\varepsilon_{k}$ выбираем, как в [10].

Вычисления заканчиваем, если

$$
\left\|x_{k+1}-x_{k}\right\| \leqslant \varepsilon_{1}=0,000001
$$

и

$$
\left|\sum_{i=1}^{m}\left(f_{i}^{2}\left(x_{k+1}\right)-f_{i}^{2}\left(x_{k}\right)\right)\right| \leqslant \varepsilon_{2}=0,0001 .
$$

Приведем таблицу числа итераций, необходимых для достижения за-

\begin{tabular}{|c|c|c|c|c|c|}
\hline \multirow{2}{*}{$\begin{array}{c}\text { Примеры и } \\
\text { исходные данные }\end{array}$} & \multicolumn{5}{|c|}{ Методы } \\
\hline & 1 & 2 & 3 & 4 & 5 \\
\hline 1. $\begin{aligned} x_{1}{ }^{0} & =3,0 \\
x_{2}{ }^{0} & =2,0\end{aligned}$ & 6 & 6 & 6 & 5 & 2 \\
\hline 2. $\begin{aligned} x_{1}{ }^{0} & =10 \\
x_{2}{ }^{0} & =20\end{aligned}$ & 8 & 18 & 8 & 23 & 6 \\
\hline 3. $\begin{array}{l}x_{1}^{0}=1 \\
x_{2}^{0}=1\end{array}$ & 13 & 33 & 18 & 7 & 5 \\
\hline 4. $\begin{array}{l}x_{1}^{0}=1 \\
x_{2}^{0}=1\end{array}$ & 15 & 59 & 47 & 17 & - \\
\hline 5. $\begin{array}{l}x_{1}{ }^{0}=1 \\
x_{2}{ }^{0}=1\end{array}$ & 48 & 260 & 80 & 23 & - \\
\hline
\end{tabular}
данной точности при использовании различных методов.

ЛИ ТЕРА Т У РА

1. V a a rma n n, O., ENSV TA Toim., Füüs. Matem., 29, № 3, 233-240 (1980).

2. В а а р м а н н О., Изв. АН ЭССР, Физ. Матем., 19, № 3, 265-274 (1970).

3. К расносельский М. А., В айникко Г. М., З аб рейко П. П., Рутиц к и й Я. Б., Сте ценко В. Я., Приближенное решение операторных уравнений, М., «Наука», 1969.

4. В а а р м анн О., Т ельг м а М., В кн.: II симпозиум по методам решения нелинейных уравнений и задач оптимизации, Хаапсалу, 4-7 июня 1981 г., Доклады и сообщения, 1, Таллин, «Валгус», 1981, с. $84-85$.

5. В а а р м а н н О., Изв. АН ЭССР, Физ. Матем., 17, № 4, 379-390 (1968).

6. Мелешко В. И., Ж. вычисл. матем. и матем. физ., 17, № 5, 1132-1143 (1977).

7. Dennis, G. E. jun., In: The State of the Art in Numerical Analysis (ed. Jacobs, D.), Academic Press, London-New York-San Francisco, 1977, p. 269306.

8. Петерсен И., Кукс Я. и др., Прикладные программы по математической статистике для ЭВМ «Минск-32», Таллин, 1977.

9. Bus, J. C. P., von Domsela a r, B., Kok, J., Math. Cent. Afd. Num. Wisk., 17, $1-42$ (i975).

10. Simmons, D. M., Nonlinear Programming for Operations Research, Englewood Cliffs, New Jersey, 1975 .

Ннститут кибернетики

Академии наук Эстонской ССР
Поступила в редакцию $22 /$ II 1982 


\section{PSEUDOPOOORDOPERAATORI APROKSIMEERIMISEL POHINEVATE ITERATSIOONIMEETODITE KOONDUVUSPIIRKONNA LAIENDAMINE}

Levinumaid vōtteid làiendada mingi iteratsioonimeetodi koonduvuspiirkonda, aga mõnikord ka selleks, et kiirendada vaadeldava meetodiga defineeritud lähislahendite koonduvust, on iteratsioonisammu pikkust reguleerivate nn. relaksatsiooniparameetrite sissetoomine iteratsioonivalemisse. Käesolevas töös (teoreem 1) on esitatud tingimused, mida peavad rahuldama relaksatsiooniparameetrid $\varepsilon_{h}$, et iteratsioonivalemid, mis pōhinevad pseudopöördoperaatori aproksimeerimisel, annaksid koonduva lähislahendite jada halvema alglähendi $x_{0}$ puhul, võrreldes juhuga. kui neis valemites $\varepsilon_{k}=1$,

Olenevalt tingimustest, mida peab rahuldama operaator $F(x)$, on saadud kas lineaarne vōi superlineaarne lähislahendite jada koonduvuskiiruse hinnang. Teoreemist 1 iäreldub lihtsalt teoreem 2, milles on toodud Levenberg-Marquardti tüüpi meetoditega määratud lähislahendite jada koonduvuse tingimused ia hinnangud selle koonduvus. kiiruse kohta. Teoreetiliste väidete illustreerimiseks on lahendatud 5 näiteülesannet.

\section{O. VAARMANN, Marika LOMP}

\section{ON ENLARGING THE DOMAIN OF CONVERGENCE OF ITERATIVE METHODS WITH APPROXIMATION PSEUDOINVERSE OPERATOR}

The problem is to solve an equation $F(x)=0$ (1) or to minimize the functional $\varphi(x)=$ $=\|F(x)\|^{2}(2)$, where $F(x)$ is a continuously Frechet differentiable operator from one Hilbert space, $H_{1}$, into another Hilbert space, $H_{2}$, and such that $F^{\prime}(x)$ has the closed range, while $F^{\prime}(x)$ need not be invertible. For finding a solution to this problem, methods based on solving the normal equation $\left[F^{\prime}(x)\right]^{*} F(x)=0(3)$ can be employed. In the present paper, particularly, iterative methods of the form $x_{h+1}=x_{h}-\varepsilon_{h} A_{h} F\left(x_{h}\right)$ $(k=0,1, \ldots)(4)$, where $A_{k}$ is an approximation to $\left[F^{\prime}\left(x_{k}\right)\right]^{+}$and $\varepsilon_{k}$ is a real value parameter suitably chosen in the interval [0.1] to guarantee the convergence of $(4)$ from a poor starting point $x_{0}$, are considered. This is a common strategy or enlarging the domain of convergence and sometimes also for convergence acceleration. Before deriving the main results of this paper which are stated in Theoremes $1-3$, several properties of the ortoprojectors associated with the pseudoinverses in Hilbert space are established. It is proved (Theorem 1) if parameters $\varepsilon_{k}$ are subject to certain constraints, $F^{\prime}(x)$ satisfies a Lipschitz condition and has the uniformly bounded pseudoinverse and certain additional (reasonable) assumptions are fulfilled in a certain bounded region $S$ of $H_{1}$, then, with the help of the formula (4). it is possible to construct a sequence of approximations that converges to a solution $x^{*} \in S$ of the equation (3). Sometimes it is true, starting from an arbitrary $x_{0} \in S$. The rapidity of convergence is estimated, in the general case by $\left\|x_{h}-x^{*}\right\| \leqslant r_{1} \delta^{h}$, where $r_{1}$ is a positive constant and $\delta \in[0,1)$ or by $\left\|x_{k}-x^{*}\right\| \leqslant r_{1} \prod_{i=0}^{h} \delta_{i}$, where $\delta_{i} \rightarrow 0$ in the limit as $i \rightarrow \infty$ if $F^{\prime}(x)$ satisfies the condition (8). Theorem 1, as applied to investigate the convergence of Levenberg-Marquardt-type methods, yields Theorem 2. Especially in the case of $\varepsilon_{k}=1 \quad(k=0,1, \ldots)$ from Theorem 2 and Theorem $\left.1{ }^{i}\right]$ one gets Theorem 3 . Finally, for illustrative purposes of above methods (4), five sample problems have been solved. 